\title{
Adapted first-line treatment of Helicobacter pylori infection in Algerian children
}

\author{
Mostefa Moubria,b, Nicolas Kalachc, Rezki Larras ${ }^{b}$, Hassina Berrah ${ }^{a, b}$, Fouzia Mouffok ${ }^{b}, Z^{2}$ or Guechib, \\ Samy Cadranel ${ }^{\mathrm{d}}$
}

Hospital N. Hamoud, CHU Hussein-Dey, Algiers, Algeria; Laboratoire Algérien de Recherche sur Helicobacter (LARH), Algiers, Algeria; Saint Vincent de Paul Hospital, Institut Catholique de Lille, Lille, France; Queen Fabiola Children's Hospital, Free University of Brussels, Belgium

Abstract

Background Helicobacter pylori (H. pylori) infection is acquired in early life and continues to have a high prevalence, especially in developing countries. Growing antibiotic-resistant strains necessitate adapted treatments. This study aimed to compare the efficacy, side effects, and influence of resistance of $H$. pylori strains between two different treatments.

Methods This prospective, randomized blind study enrolled 112 symptomatic children infected with $H$. pylori (66 girls, mean age 11.1 years). Treatments, allocated randomly irrespective of the susceptibility of the strains, were either the standard omeprazole-amoxicillin-clarithromycin combination for 7 days (OAC7; group A) or omeprazole-amoxicillin with a higher dose of metronidazole (40 instead of $20 \mathrm{mg} / \mathrm{kg} / \mathrm{d}$ ) for 10 days (OAM10; group B).

Results Before treatment, the resistance rates of $H$. pylori strains to metronidazole or clarithromycin were $37 \%$ and $13 \%$, respectively, with $7 \%$ resistant to both antibiotics and neither to amoxicillin. Eradication rates obtained with OAM10 ( $80 \%$ by intention-to-treat [ITT] and $88 \%$ by per protocol $[\mathrm{PP}]$ analysis) were higher than with OAC7 (68\% in ITT and $71 \% \mathrm{PP})$ and the differences (12\% in ITT and $17 \% \mathrm{PP})$ were statistically significant $(\mathrm{P}=0.03)$. Successful treatments with OAM10 were obtained in metronidazole resistant strains and were more effective in children aged $>10$ years ( $\mathrm{P}=0.02$ by ITT and $\mathrm{P}=0.04$ by $\mathrm{PP}$ ). Only light or moderate side effects, mainly digestive, were observed.

Conclusion Because of its therapeutic efficacy, good tolerance and lower cost the OAM10 can be considered as an appropriate first-line therapeutic scheme in Algeria.

Keywords Helicobacter pylori, children, first-line eradication treatment, antibiotic resistance

Ann Gastroenterol 2019; 32(7): 1-7

${ }^{a}$ Department of Paediatrics, Hospital N. Hamoud, CHU HusseinDey, Algiers, Algeria (Mostefa Moubri, Hassina Berrah); ${ }^{\text {L Laboratoire }}$ Algérien de Recherche sur Helicobacter (LARH), Algiers, Algeria (Mostafa Moubri, Rezki Larras, Hassina Berrah, Fouzia Mouffok, Zhor Guechi); 'Saint Vincent de Paul Hospital, Institut Catholique de Lille (GH-ICL), Lille, France (Nicolas Kalach); ${ }^{\mathrm{D}}$ Department of Gastroenterology, Queen Fabiola Children's Hospital, Free University of Brussels, Belgium (Zhor Guechi)

Conflict of Interest: None

Correspondence to: Samy Cadranel, Department of Gastroenterology, Queen Fabiola Children's Hospital, Free University of Brussels, Belgium, e-mail: scadrane@ulb.ac.be

Received 12 February 2018; accepted 26 July 2018; published online: 3 October 2018

DOI: https://doi.org/10.20524/aog.2018.0317

\section{Introduction}

Helicobacter pylori (H. pylori) infection plays an important epidemiological role as one of the most prevalent chronic infections, with more than $50 \%$ of the world's population being infected. A prevalence of over $80 \%$ has been found in developing countries [1], whereas in industrial nations, a much lower and declining prevalence is usually reported [2]. These differences are thought to be due to several environmental factors, such as different infectious mechanisms, better hygienic and social conditions and, as an additional factor, the excessive use of antibiotics in the treatment of several bacterial infections [3-7], with the worrying negative consequence of an increased resistance of $H$. pylori strains $[8,9]$. The infection occurs mainly during childhood $[10,11]$ and persists throughout life $[12,13]$.

$H$. pylori infection is an important cause of ulcerations of the stomach and duodenum, not only in adults [14] but also in 
children [15]. It can also cause, mainly in adulthood, atrophy of the gastric mucosa and intestinal metaplasia responsible for gastric malignancies and mucosa-associated lymphoid tissue (MALT) lymphoma [16].

In Algeria an infection rate of $82 \%$ was found in 2006 in 275 symptomatic children with a mean age of 8.4 years [17], whereas in 2008 a study by Guechi and the Algerian Helicobacter Research Laboratory confirmed the early acquisition of $H$. pylori infection in Algerian schoolchildren with a seroprevalence of $38 \%$, increasing with age to 57\% [18]. The ESPGHAN-NASPGHAN evidence-based guidelines [19] and their 2016 update [20] insist that local conditions must be taken into account in order to achieve successful results from eradication treatments.

The primary goal of the present study, performed before the publication of the ESPGHAN-NASPGHAN guidelines, was to evaluate, in $H$. pylori-infected symptomatic children, the efficacy and tolerance of two first-line eradication treatments and to investigate factors influencing the efficacy of the treatments, mainly the resistance of $H$. pylori strains. Because some specific conditions are still lacking in our country, a secondary goal was to assess the efficacy, sensitivity and specificity of noninvasive diagnostic tools compared to invasive methods.

\section{Patients and methods}

In order to test the efficacy of different treatment regimens, a prospective randomized study was carried out from May 2006 to March 2010. The study population included 160 consecutive symptomatic children aged 5-16 years who underwent an upper gastrointestinal (GI) endoscopy for several indications, including chronic abdominal pain, chronic dyspepsia (vomiting, nausea, excessive belching, halitosis), history of upper GI bleeding, unexplained iron deficiency and growth retardation. Exclusion criteria were diabetes, severe chronic kidney, heart or liver disease, treatment with nonsteroidal antiinflammatory drugs, previous treatment for $H$. pylori infection, and known GI diseases such as gastroesophageal reflux, active GI bleeding, celiac disease and inflammatory bowel diseases. H. pylori infection was based on several diagnostic tests, using gastric biopsies processed with a rapid urease test (RUT), the Pronto-Dry ${ }^{\circledast}$ (Medical Instruments Corporation, Lyon, France), and histology and culture with antimicrobial susceptibility testing for the determination of the sensitivity of $H$. pylori strains. Noninvasive diagnostic tests, including the rapid $H$. pylori monoclonal stool antigen test (HpStAR) (DakoCytomation Ltd., Liverpool, United Kingdom) and the non-radioactive ${ }^{13} \mathrm{C}$ urea breath test $\left({ }^{13} \mathrm{C} \mathrm{UBT}\right)$, were also used. The diagnosis of $H$. pylori infection was based on the positivity of the culture and/or histology confirmed by the ${ }^{13} \mathrm{C}$ UBT. Only the patients who fulfilled these criteria were included.

The treatment protocol was explained to the parents and the children and their informed consent was obtained before enrolment. Randomization of treatment was done using the sorting method of closed envelopes where the treatment was attributed randomly in clusters of 4 . Infection by $H$. pylori strains was found in 112/160 (70\%) children, 66 girls and
45 boys aged 5.2 to 15.9 years (mean age 11.1 years). After randomization, patients were allocated to one of the two treatment schemes. Children in group A received for 7 days the classical treatment of the combination of omeprazoleamoxicill in'larithromycin (OAC7), adjusted for the child's bodyweight: in children heavier than $30 \mathrm{~kg}$ (usually 10 years old) it consisted of omeprazole $2 \times 20 \mathrm{mg} / \mathrm{d}$, amoxicillin $50 \mathrm{mg} / \mathrm{kg} / \mathrm{d}$ b.i.d. with a maximum of $2 \mathrm{~g} / \mathrm{d}$ and clarithromycin $15 \mathrm{mg} / \mathrm{kg} / \mathrm{d}$ b.i.d. The dose of omeprazole was reduced to $2 \times 10 \mathrm{mg} / \mathrm{d}$ in children below $30 \mathrm{~kg}$ bodyweight. Children in group B received for 10 days the alternative treatment (OAM10) of omeprazole $2 \times 20 \mathrm{mg} / \mathrm{d}$ with amoxicillin $50 \mathrm{mg} / \mathrm{kg} / \mathrm{d}$ b.i.d. with a maximum of $2 \mathrm{~g} / \mathrm{d}$ and metronidazole $40 \mathrm{mg} / \mathrm{kg} / \mathrm{d}$ b.i.d. with a maximum of $1.5 \mathrm{~g} / \mathrm{d}$ in children above $30 \mathrm{~kg}$ and $1 \mathrm{~g} / \mathrm{d}$ in children below $30 \mathrm{~kg}$ bodyweight. The dose of omeprazole was reduced to $2 \times 10 \mathrm{mg} / \mathrm{d}$ in the same way as in the OAC7. Side effects could be reported at any time to the prescribing physician and withdrawal from the study was granted. Patients were seen at the end of the treatment period to collect data on side effects and evaluate their compliance by recounting the tablets. The $H$. pylori status was assessed 8-12 weeks after the termination of the treatment by a second upper GI endoscopy with biopsies processed for RUT, histology and culture, a ${ }^{13} \mathrm{C}$ UBT and an HpStAR. Eradication of the $H$. pylori infection was attested by the negativity of all or $4 / 5$ diagnostic tests, exempting the culture because of its high specificity. In case of treatment failure, attested by the positivity of all tests or at least positivity of the culture or histology + RUT, a rescue treatment was proposed.

\section{Statistical analysis}

The statistical analysis was performed using STATISTICA for Windows (Epi. Info, Atlanta USA) version 6.04. To test the statistically significant differences of two nominally distributed factors the $\chi^{2}$ test (chi-square test) or Fisher test was used, whereas the Student's $t$-test was used for comparing mean values. The differences were considered significant for values of $\mathrm{P} \leq 0.05$. Results expressed as "per protocol" (PP) are based on data obtained in patients who fulfilled all the items of the protocol, whereas results expressed as "intention to treat" (ITT) are based on data obtained from all the enrolled patients even if they did not complete all the items. The eradication rate was calculated with a confidence interval (CI) of $95 \%$.

\section{Results}

Seven of the 112 recruited subjects were lost to follow up (5 in the OAM10 group and 2 in the OAC7 group) so that eventually data from 105 children were analyzed. The most common endoscopic finding (Table 1) in H. pylori-infected children was antral nodularity $(76,68 \%)$, whereas duodenal ulcer or erosions were observed in 5 children (4.5\%).

Treatment compliance was evaluated according to the quantity of medication taken and considered as "good" in 98/105 (93\%) patients, who took $100 \%$ of the medication, and as "average" 
in the $2 / 105$ patients who took only $75 \%$. None of them was considered as "poor" (taking less than $75 \%$ of the medication).

Successful eradication of the infection was attained in 83 children, with an overall eradication rate of $74 \%$ by ITT and $79 \%$ by PP. Higher eradication rates were obtained with OAM10 than with OAC7, since treatment was successful in $44 / 55(80 \%)$ by ITT and $44 / 50(88 \%)$ by PP in the OAM 10 group, and in $39 / 57$ (68\%) by ITT and $39 / 55$ (71\%) by PP in the OAC7 group. These differences were statistically significant for the PP $(\mathrm{P}<0.03)$ but not for the ITT values (Table 2$)$.

There were important differences according to the resistance or sensitivity of the $H$. pylori strains: respectively $51 \%$ and $65 \%$ by ITT and PP for antibiotic-resistant $H$. pylori strains compared to $75 \%$ and $91 \%$ by ITT and PP for antibioticsensitive strains $(\mathrm{P}<0.03$ and $\mathrm{P}<0.01$, respectively) (Table 3 ).

The eradication rates in relation to clarithromycin or metronidazole resistance were analyzed in $31 \mathrm{H}$. pylori strains in the OAM10 group and in 28 strains in the OAC7 group. In the OAM10 group the eradication rate was similar whether the $H$. pylori strains were metronidazole-resistant or not ( $90 \%$ vs. $91 \%, \mathrm{P}=0.67$ ) whereas a difference, although non-significant,

Table 1 Endoscopic findings

\begin{tabular}{lcc}
\hline Endoscopic aspect & $\mathrm{N}$ & $\%$ \\
\hline Normal & 09 & 8 \\
Nodularity & 76 & 68 \\
Enlarged gastric folds & 01 & 0.9 \\
Erosive duodenitis & 03 & 2.7 \\
Duodenal ulcer & 02 & 1.8 \\
\hline
\end{tabular}

Table 2 Eradication rates according to different therapeutic schemes

\begin{tabular}{lccccccc} 
Scheme & \multicolumn{3}{c}{ PP } & & \multicolumn{3}{c}{ ITT } \\
\cline { 2 - 4 } \cline { 6 - 8 } & $\mathrm{n}$ & $\%$ & $\mathrm{P}$ & $\mathrm{n}$ & $\%$ & $\mathrm{P}$ \\
\hline OAC 7 & $39 / 55$ & 71 & & $39 / 57$ & 68 & \\
& & & & & & \\
OAM 10 & $44 / 50$ & 88 & $0.031^{*}$ & & $44 / 55$ & 80 & 0.161 NS \\
Total & $83 / 105$ & 79 & & $83 / 112$ & 74 & \\
\hline
\end{tabular}

${ }^{\star}$ According to $\chi^{2}$ test; $\mathrm{P}$ was considered significant when $<0.05$

NS, not significant; OAC, omeprazole-amoxicillin-clarithromycin; OAM, omeprazole-amoxicillin-metronidazole; PP, per protocol; ITT, intention to treat was observed for clarithromycin sensitivity or resistance (93\% vs. $67 \%, \mathrm{P}=0.27$ ) (Table 4 ). In the OAC7 group, eradication rates were lower for metronidazole-resistant $H$. pylori strains than for sensitive $H$. pylori strains, although the difference was not statistically significant ( $50 \%$ vs. $78 \%, P=0.30$ ). In contrast, eradication rates differed dramatically between clarithromycinresistant and clarithromycin-sensitive $H$. pylori strains ( $83 \%$ vs. $0 \%, \mathrm{P}<0.01$ ) (Table 5).

Other factors studied concerned age and sex. A higher eradication rate was observed in children older than 10 years by both ITT ( $81 \%$ vs. $62 \%, \mathrm{P}<0.02)$ and PP ( $85 \%$ vs. $68 \%, \mathrm{P}<0.04)$ analysis. Better results, but without statistical significance, were observed in boys than in girls by ITT ( $80 \%$ vs. $70 \%, \mathrm{P}=0.20)$ and PP ( $86 \%$ vs. $74 \%, \mathrm{P}=0.14$ ), although the antibiotic resistance of $H$. pylori strains did not differ significantly between boys and girls $(\mathrm{P}=0.85)$. The compliance was overall good, being average in only $5 / 105$ patients, but this did not statistically influence the results ( $75 \%$ vs. $33 \%$ by ITT, $\mathrm{P}=0.16$, and $80 \%$ vs. $50 \%$ by $\mathrm{PP}, \mathrm{P}=0.37$ ).

Because noninvasive diagnostic tests are not available in many developing regions of the world, the secondary goal of this study was to compare the different noninvasive diagnostic tests with the invasive RUT, histology and culture performed in all patients (Table 6).

Side effects were evaluated in all 112 children recruited, including the 105 who completed the program and the 7 who did not undergo the second upper GI endoscopy. Mild to moderate, mainly GI symptoms were reported in 44 patients, $(80.4 \%$ by ITT and $79.5 \%$ by PP). In the OAM10 group, side effects were reported in 13 children (24\%) and consisted of abdominal cramps or diarrhea in 12 patients and skin rash in one. In the OAC7 group, side effects were reported in 33 children (58\%), including digestive symptoms in 25 children (75\%) and a bitter or metallic taste, probably due to clarithromycin, in 8 (25\%).

The cost of the treatment courses, calculated according to the number and cost of the tablets ingested, amounted to $€ 42$ for OAC7 and $€ 39$ for OAM10 but could probably be reduced by using generic drugs.

\section{Discussion}

There are few randomized controlled trials of $H$. pylori eradication in children and none in Algeria. During the nineties, eradication rates of $90 \%$ were reported, but the

Table 3 Eradication rate with different therapeutic regimens depending on Helicobacter pylori antimicrobial susceptibility testing ( $\mathrm{n}=28$ )

\begin{tabular}{lcccc}
\hline Susceptibility & Eradication with OAM10 n (\%) & Eradication with OAC7 n (\%) & Total & P \\
\hline Metronidazole-susceptible strains & $17(89.5)$ & $14(77.8)$ & 31 & $0.034^{*}$ \\
Metronidazole-resistant strains & $11(91.5)$ & $5(50)$ & 16 & 45 \\
Clarithromycin-susceptible strains & $26(93)$ & $19(83)$ & $0.001^{*}$ \\
Clarithromycin-resistant strains & $2(66.5)$ & $0(0)$ & 2 & \\
\hline${ }^{*}$ According to Fischer’s exact test; P was consiger significant
\end{tabular}

${ }^{*}$ According to Fischer's exact test; P was considered significant when $<0.05$

OAC, omeprazole-amoxicillin-clarithromycin; OAM, omeprazole-amoxicillin-metronidazole 
Table 4 Eradication rate with OAM10 and Helicobacter pylori strains according to susceptibility testing $(\mathrm{n}=31)$

\begin{tabular}{|c|c|c|c|c|}
\hline OAM10 & Metronidazole-susceptible strains n (\%) & Metronidazole-resistant strains n (\%) & Total & $\mathrm{P}^{*}$ \\
\hline Eradication & $17(89.5)$ & $11(91.5)$ & 28 & \multirow{3}{*}{$\begin{array}{c}0.672 \\
\text { NS }\end{array}$} \\
\hline Failed & $2(10.5)$ & $1(8.5)$ & 3 & \\
\hline Total & $19(100)$ & $12(100)$ & 31 & \\
\hline OAM10 & Clarithromycin-susceptible strains n (\%) & Clarithromycin-resistant strains n (\%) & Total & \\
\hline Eradication & $26(93)$ & $2(66.5)$ & 28 & \multirow{3}{*}{$\begin{array}{c}0.271 \\
\text { NS }\end{array}$} \\
\hline Failed & $2(7)$ & $1(33.5)$ & 3 & \\
\hline Total & $28(100)$ & $3(100)$ & 31 & \\
\hline
\end{tabular}

${ }^{\star}$ According to Fischer's exact test; P was considered significant when $<0.05$

NS, not significant; OAM, omeprazole-amoxicillin-metronidazole

Table 5 Eradication rate with OAC7 and Helicobacter pylori strains according to susceptibility testing $(\mathrm{n}=28)$

\begin{tabular}{lccc}
\hline OAC7 & Metronidazole susceptible-strains n (\%) & Metronidazole resistant-strains n (\%) & Total \\
\hline Eradication & $14(77.8)$ & $5(50)$ & 19 \\
Failed & $4(22.2)$ & $5(50)$ & 9 \\
Total & $18(100)$ & $10(100)$ & 28 \\
\hline OAC7 & Clarithromycin susceptible-strains n (\%) & Clarithromycin resistant-strains n (\%) & Total \\
\hline Eradication & $19(83)$ & $0(0)$ & 19 \\
Failed & $4(17)$ & $5(100)$ & 9.001 \\
Total & $23(100)$ & $5(100)$ & 28 \\
\hline
\end{tabular}

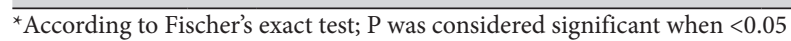

NS, not significant; OAC, omeprazole-amoxicillin-clarithromycin

Table 6 Comparative assessment of the different diagnostic tools

\begin{tabular}{lcccccc}
\hline Tests & $\mathrm{n}$ & Efficacy \% & Sensitivity (\%) & Specificity (\%) & Positive predictive value (\%) & Negative predictive value (\%) \\
\hline UBT & 112 & 97 & 97.3 & 95.6 & $98.2(93.0-99.7)$ & $93.5(81.1-98.3)$ \\
RUT & 112 & 97 & 95.5 & 100 & $100(95.7-100)$ & $90.6(78.6-96.5)$ \\
Histology & 109 & 96 & 99.1 & 89.4 & $95.6(89.6-98.4)$ & $97.7(86.2-99.9)$ \\
Stool Ag & 110 & 96 & 93.6 & 100 & $100(95.5-100)$ & $87.3(74.9-94.3)$ \\
Serology & 103 & 82 & 85.4 & 74.4 & $88.9(80.6-94.1)$ & $68.1(52.7-80.5)$ \\
Culture & 110 & 77 & 67.3 & 100 & $100(93.9-100)$ & $57.1(45.9-67.7)$ \\
\hline
\end{tabular}

UBT, urea breath test; RUT, rapid urease test

efficacy of these first-line treatments, which combine a proton pump inhibitor with two antibiotics, has notably decreased to $70-74 \%$ [21], mainly as a result of primary antibiotic resistance of $H$. pylori strains [22,23] or the lack of therapeutic adherence $[24,25]$. The most widely used therapeutic scheme in the western world, based on the combination of the proton pump inhibitor omeprazole with 2 antibiotics, amoxicillin and clarithromycin, for 7 days, reaches only a rather disappointing eradication rate of $71 \%$ by PP and $68 \%$ by ITT analysis [26].

In a previous study [27], based on a combination of omeprazole with amoxicillin and a higher dose than usual of metronidazole, we obtained eradication rates of $78 \%$ with the 7 -day course and $86 \%$ with the 10 -day course, considerably higher than those achieved with the classical normal dose of metronidazole. However, the series of patients was too small to allow the $8 \%$ difference between the longer and the shorter courses to reach statistical significance.

In the present study, better results were obtained with our double dose of metronidazole during a longer course of 10 days, compared to the classical OAC7 ( $\mathrm{P}<0.03$ by PP). Higher eradication rates were obtained using the double dose of metronidazole in OAM for 10 days in the present study, in comparison with other schemes using OAM 7 days $[24,28,29]$, the regular $20 \mathrm{mg} / \mathrm{kg}$ dose of OAM over 14 days [30], and our previous OAM 7-day study with a high dose of metronidazole (Table 7). Two factors, a higher metronidazole dose $(40 \mathrm{mg} / \mathrm{kg}$ instead of $20 \mathrm{mg} / \mathrm{kg}$ ), but also prolongation of the treatment from 7 days to 10 days, seem to have favorably influenced 
Table 7 Eradication rates with different doses and durations of OAM treatments

\begin{tabular}{|c|c|c|c|}
\hline Author & Year & OAM/days & Eradication rates \\
\hline \multicolumn{4}{|c|}{ Faber [27] N=57 } \\
\hline Israel & 2005 & 7 & 73.4 \\
\hline \multicolumn{4}{|c|}{ Oderda [23] N=388 } \\
\hline Italy & 2007 & 7 & 66 \\
\hline \multicolumn{4}{|c|}{ Nguyen [28] N=117 } \\
\hline Vietnam & 2008 & 7 & 62.1 \\
\hline \multicolumn{4}{|c|}{ Moubri [26] N=23 } \\
\hline Algeria & 2008 & $7^{*}$ & 78 \\
\hline \multicolumn{4}{|c|}{ Chen [29] N=35 } \\
\hline China & 2004 & 14 & 77 \\
\hline \multicolumn{4}{|c|}{ Present study $\mathrm{N}=50$} \\
\hline Algeria & 2010 & $10^{*}$ & $\begin{array}{c}88 \text { PP N}=50 \\
80 \text { ITT N }=55\end{array}$ \\
\hline
\end{tabular}

the outcome to reach almost the acceptable recommended eradication rate of $90 \%$ [19]. The most recent recommendations specify that the shorter 7-day treatment scheme should be abandoned in favor of the longer 14-day regimen [20]. However, apart from its higher cost, it may be difficult, in many day-to-day clinical contexts, to obtain good adherence to a 2-week-long duration of treatment.

Drug dosage, treatment duration and patients' adherence to therapy are factors that influence the outcome of treatment, but even more important is the growing resistance of H. pylori strains [22-23], especially to clarithromycin and nitroimidazoles, depending on the regional consumption of these drugs [9]. Over 50\% resistance to metronidazole has been reported in developing countries [31]. Although resistance to metronidazole is rather stable, an increasing number of $H$. pylori strains are resistant to both macrolides and nitroimidazoles.

This double resistance still remains low in children, but raises major concern and accounts for the treatment failures of most therapeutic schemes [32]. Resistance to other antibiotics such as quinolones is also increasing [33], whereas resistance to amoxicillin is exceptionally rare and was not seen in our study. In regions where the resistance to clarithromycin is higher than 15-20\%, the Maastricht V consensus [34] and recent pediatric guidelines recommend using this antibiotic for the treatment of $H$. pylori infections only after testing the resistance of the $H$. pylori strains $[19,20]$. Adaptation of the treatment according to the resistance of $H$. pylori strains allows treatment failures to be reduced. Treatment strategies need to be tailored according to the national, regional or even local antibiotic resistance of H. pylori strains. The data available in Algeria [27] show a high prevalence of metronidazole-resistant $H$. pylori strains in the general population, although, as reported elsewhere, it has been decreasing in the pediatric population [35]. In contrast, our personal data show a steady increase in clarithromycin resistance from 5\% in 2002 to $13 \%$ in 2010, probably due to an increasing use of this antibiotic for other infections [33]. In our study, the results achieved with the OAM10 scheme with a high dose of metronidazole were superior to results reported in the literature $[24,26]$.

The sensitivity of $H$. pylori strains strongly influences the efficacy of the antibiotic treatments in the developed [24] as well as in the developing [36] world. Because of the high prevalence of resistant $H$. pylori strains in children, first-line treatment should be started only after a strain's resistance to antibiotics has been tested using the classical antimicrobial susceptibility testing method or molecular polymerase chain reaction, when these techniques are available [19]. Since such techniques are either unavailable or too expensive in Algeria, and considering our results, the OAM10 scheme can be considered as an interesting first line treatment in children.

Compared to rates reported in the literature with OAM7 and OAM14, the eradication rates obtained with the high-dose metronidazole OAM 10-day triple therapy in the present study were $21 \%$ and $16 \%$ higher, respectively [24], while compared to OAC7 in the present study, gains of $17 \%$ by PP and $12 \%$ by ITT were observed. These better results are probably attributable to a moderate, acceptable extension of the treatment duration, but mainly to the higher dosage of metronidazole.

In the HOMER study in adults, Bardhan et al showed that a higher dose of metronidazole resulted in better global eradication rates, including in metronidazole-resistant strains of $H$. pylori [37]. A similar effect was observed in the present study, where the higher dose was associated with a rise in eradication rate from $71 \%$ to $88 \%(\mathrm{P}=0.03)$, not so far from the recommended $90 \%$. Poor therapeutic adherence is an important factor in treatment failure [25]. Parents and children should be instructed about the importance of respecting the timing and dose of medication and alerted about possible side effects that, wrongly interpreted, could induce poor compliance or even premature cessation of the treatment.

In the present study, performed before the publication of the first ESPGHAN-NASPGHAN consensus guidelines, better treatment efficacy was observed in children older than 10 years. Familial density and promiscuity in younger children, with more frequent reinfections, may account for the significant differences ( 0.04 by PP, 0.02 by ITT). Side effects were reported significantly $(\mathrm{P}=0.0004)$ more frequently in the OAC7 group (58\%) than in the OAM 10 group (24\%), although no treatment had to be stopped. The OAM10 triple therapy appeared to be better tolerated, despite the high dose of metronidazole and the longer duration of the treatment.

In conclusion, in Algeria, the triple therapy combining omeprazole, amoxicillin and a double dose of metronidazole for 10 days can be recommended as first-line treatment in $H$. pylori-infected children, since it shows better efficacy than the standard 7-day treatment with the association of OAC, its better tolerance and relatively cheaper cost. Because bismuth salts are still and wrongly unavailable in our country, re-treatment of eradication failures requires either a 14-day scheme, according to carefully tailored antimicrobial susceptibility testing, or sequential therapy. 


\section{Summary Box}

\section{What is already known:}

- Helicobacter pylori (H. pylori) infection is acquired in early life and has a high prevalence in developing regions

- There is growing antibiotic resistance to H. pylori strains

- Eradication treatments can yield unsatisfactory results

\section{What the new findings are:}

- There is a high prevalence of resistance to clarithromycin and metronidazole in Algeria

- The poor results with the classical first-line treatment were confirmed

- Good results were obtained using a high dose of metronidazole, including in resistant $H$. pylori strains, with relatively few side effects

\section{Acknowledgment}

The authors wish to acknowledge the memory of late Professor Brahim Touchene, who founded and directed the LARH. He was an essential promotor of many research studies in the field of H. pylori, including the $\mathrm{PhD}$ thesis on which the present study is based. His generous guidance is fondly remembered.

\section{References}

1. Moosazadeh M, Lankarani KB, Afshari M. Meta-analysis of the prevalence of Helicobacter pylori infection among children and adults of Iran. Int J Prev Med 2016;7:48.

2. Asgeirsdottir GA, Kjartansdottir I, Olafsdottir AS, et al. Helicobacter pylori infection in Icelandic children. Scand J Gastroenterol 2017;52:686-690.

3. Vale FF, Vitor JM. Transmission pathway of Helicobacter pylori: does food play a role in rural and urban areas? Int J Food Microbiol 2010;138:1-12.

4. Daugule I, Rowland M. Helicobacter pylori infection in children. Helicobacter 2008;13 Suppl 1:41-46.

5. Rothenbacher D, Bode G, Adler G, Brenner H. History of antibiotic treatment and prevalence of $H$. pylori infection among children: results of a population-based study. J Clin Epidemiol 1998;51:267-271.

6. Daugule I, Rumba I, Lindkvist P, Bergström M, Ejderhamn J. A relatively low prevalence of Helicobacter pylori infection in a healthy paediatric population in Riga, Latvia: a cross-sectional study. Acta Paediatr 2001;90:1199-1201.

7. Malaty HM. Helicobacter pylori infection and eradication in paediatric patients. Paediatr Drugs 2000;2:357-365.

8. Megraud F. Resistance of Helicobacter pylori to antibiotics. Aliment
Pharmacol Ther 1997;11(Suppl 1):43-53.

9. Koletzko S, Richy F, Bontems P, et al. Prospective multicentre study on antibiotic resistance of Helicobacter pylori strains obtained from children living in Europe. Gut 2006;55:1711-1716.

10. Ahmed KS, Khan AA, Ahmed I, et al. Prevalence study to elucidate the transmission pathways of Helicobacter pylori at oral and gastroduodenal sites of a South Indian population. Singapore Med J 2006;47:291-296.

11. Kivi M, Tindberg Y. Helicobacter pylori occurrence and transmission: a family affair? Scand J Infect Dis 2006;38:407-417.

12. Czinn SJ. Helicobacter pylori infection: detection, investigation, and management. J Pediatr 2005;146:S21-S26.

13. Lehours P, Yilmaz O. Epidemiology of Helicobacter pylori infection. Helicobacter 2007;12(Suppl 1):1-3.

14. Blaser MJ. Helicobacter pylori and the pathogenesis of gastroduodenal inflammation. J Infect Dis 1990;161:626-633.

15. Kalach N, Bontems P, Koletzko S, et al. Frequency and risk factors of gastric and duodenal ulcers or erosions in children: a prospective 1-month European multicenter study. Eur J Gastroenterol Hepatol 2010;22:1174-1181.

16. Forman D, Newell DG, Fullerton F, et al. Association between infection with Helicobacter pylori and risk of gastric cancer: evidence from a prospective investigation. BMJ 1991;302:1302-1305.

17. Moubri M, Larras R, Berrah H, Moufok F, Guechi Z, Touchene B. Helicobacter pylori infection in Algerian children; about 275 cases. Helicobacter 2006;11:378.

18. Guechi Z, Riad H, Naitkaci S. Séroprévalence de l'infection à Helicobacter pylori dans l'algérois. Journal Algérien de Médecine 2008;16:12-14.

19. Koletzko S, Jones NL, Goodman KJ, et al; H pylori Working Groups of ESPGHAN and NASPGHAN. Evidence-based guidelines from ESPGHAN and NASPGHAN for Helicobacter pylori infection in children. J Pediatr Gastroenterol Nutr 2011;53:230-243.

20. Jones NL, Koletzko S, Goodman K, et al. Joint ESPGHAN/ NASPGHAN guidelines for the management of Helicobacter pylori in children and adolescents. J Pediatr Gastenterol Nutr 2017;64:991-1003.

21. Iwańczak BM, Borys-Iwanicka A, Biernat M, Gościniak G. Assessment of sequential and standard triple therapy in treatment of Helicobacter pylori infection in children dependent on bacteria sensitivity to antibiotics. Adv Clin Exp Med 2016;25:701-708.

22. Serrano CA, Leon MA, Palma C, Vera M, Hernandez C, Harris PR. Helicobacter pylori-clarithromycin resistance in symptomatic pediatric patients in a high prevalence country. $J$ Pediatr Gastroenterol Nutr 2017;64:e56-e60.

23. Regnath $T$, Raecke $O$, Enninger A, Ignatius R. Increasing metronidazole and rifampicin resistance of Helicobacter pylori isolates obtained from children and adolescents between 2002 and 2015 in southwest Germany. Helicobacter 2017;22.

24. Oderda G, Shcherbakov P, Bontems P, et al; European Pediatric Task Force on Helicobacter pylori. Results from the pediatric European register for treatment of Helicobacter pylori (PERTH). Helicobacter 2007;12:150-156.

25. Kotilea K, Mekhael J, Salame A, et al. Eradication rate of Helicobacter pylori infection is directly influenced by adherence to therapy in children. Helicobacter 2017;22.

26. Khurana R, Fischbach L, Chiba N, et al. Meta-analysis: Helicobacter pylori eradication treatment efficacy in children. Aliment Pharmacol Ther 2007;25:523-536.

27. Moubri M, Larras R, Berrah H, Mouffok F, Bouhadef A, Touchene B. Traitement par le régime oméprazole-amoxicilline-métronidazole de l'infection à Helicobacter pylori chez l'enfant algérien: 7 jours vs 10 jours. Journal du Pédiatre Belge 2008;10:18-20.

28. Faber J, Bar-Meir R B, Schlesinger Y, et al. Treatment regimens for Helicobacter pylori infection in children: is in vitro susceptibility testing helpful? J Pediatr Gastroenterol Nutr 2005;40:571-574. 
29. Nguyen TV, Bengtsson C, Nguyen GK, et al. Evaluation of two triple-therapy regimens with metronidazole or clarithromycin for the eradication of $H$. pylori infection in Vietnamese children: a randomized, double-blind clinical trial. Helicobacter 2008;13:550-556.

30. Chen X, Ou B, Wu X, et al. Long-term therapeutic effect of triple therapy consisted of omeprazole, clarithromycin and amoxicillin in children with Helicobacter pylori infection and approach to retreatment after failure of the treatment. Zhonghua Er Ke Za Zhi 2004;42:417-420.

31. Vilaichone RK, Ratanachu-Ek T, Gamnarai P, et al. Extremely high prevalence of metronidazole-resistant Helicobacter pylori strains in mountain people (Karen and Hmong) in Thailand. Am J Trop Med Hyg 2016;94:717-720.

32. Schwarzer A, Urruzuno P, Iwańczak B, et al; ESPGHAN Working Group on Helicobacter pylori Infection. New effective treatment regimen for children infected with a double-resistant Helicobacter pylori strain. J Pediatr Gastroenterol Nutr 2011;52:424-428.
33. Miendje Deyi VY, Deyi VY, Bontems P, et al. Multicenter survey of routine determinations of resistance of Helicobacter pylori to antimicrobials over the last 20 years (1990 to 2009) in Belgium. J Clin Microbiol 2011;49:2200-2209.

34. Malfertheiner P, Megraud F, O'Morain CA, et al; European Helicobacter and Microbiota Study Group and Consensus panel. Management of Helicobacter pylori infection-the Maastricht V/ Florence Consensus Report. Gut 2017;66:6-30.

35. Kalach N, Serhal L, Asmar E, et al. Helicobacter pylori primary resistant strains over 11 years in French children. Diag Microbiol Infect Dis 2007;59:217-222.

36. Queiroz DM, Dani R, Silva LD, et al. Factors associated with treatment failure of Helicobacter pylori infection in a developing country. J Clin Gastroenterol 2002;35:315-320.

37. Bardhan K, Bayerdörffer E, Veldhuyzen Van Zanten SJ, et al. The HOMER Study: the effect of increasing the dose of metronidazole when given with omeprazole and amoxicillin to cure Helicobacter pylori infection. Helicobacter 2000;5:196-201. 